\title{
Real-Time Implementation of Continuous Model Based Sliding Mode Control Technique for Trajectory Tracking Control of Mobile Robot
}

\author{
A. Dumlu, and M. R. Yıldırım
}

\begin{abstract}
In this study, real-time trajectory tracking control of an autonomous mobile robot has been designed, analyzed and studied. Two control techniques such as the proportionalintegral-derivative (PID) control and model based sliding mode control (SMC) have been considered to increase the tracking performance of the mobile robot. Firstly, kinematic and dynamic analysis of the system have been obtained and then this dynamic model has been used for proposed sliding mode controller in order to increase trajectory tracking performance of the system. The experimental outcomes strongly verified that the proposed controller gives a quite well trajectory tracking response and smaller magnitude overshot compared with the classical PID controller.
\end{abstract}

Index Terms- PID control, Robot control, Sliding mode control, Trajectory control.

\section{INTRODUCTION}

$\mathrm{T}$ HE PURPOSE of robotic systems used in today's modern industrial world is to improve the quality of products, industrial productivity, correctness, speed and flexibility. Although the requirements of industrial applications are complex and difficult, robotic systems are increasingly being used more and more in dangerous, tedious or repetitive industrial processes, where people do not want to work. More efficient and quality manufacturing is an important issue of the industry, and this demand has led to the development of more skilled and modern robotic systems. These skilled systems are usually autonomous and therefore require initial actions, such as calibration, trajectory planning in order to fulfill their assigned tasks. For this reason, it is a very important step to accurately and precisely control the system in the working environment in order to be able to fulfill the assigned tasks successfully.

\footnotetext{
A.DUMLU, is with Department of Electrical Engineering University of Erzurum Technical University, Erzurum, Turkey, (e-mail: ahmetdumlu@erzurum.edu.tr).
}

M. R. YILDIRIM, is with Company of Turk Telekom, Erzurum, Turkey, (e-mail: mehmetrasimyildirim@gmail.com ).

Manuscript received September 13, 2018; accepted October 24, 2018. DOI: $10.17694 /$ bajece. 459568
The main areas of research in the field of robotics systems can be summarized as system design, trajectory planning, trajectory optimization, and motion control.

Among these scientific research areas, the control of robotic systems has a crucial place to follow a precise and reliable trajectory between the initial and final position of the robot [1]. Accurate trajectory control is vital issue for the efficient operation of a given robotic application. For this reason, a large number of control methods have been applied in the literature for various types of robotic systems, such as, $\mathrm{H}$ infinity control [2], neural network control [3], [4], adaptive fuzzy control [5], [6] and fractional order control [7-9].

Another robust control technique used for trajectory tracking control of nonlinear system in literature is SMC method. SMC method is an effective and robust control technique and has a variable structure for the unknown dynamics of unknown loads and nonlinear systems. This control method is also used to simplify design, increase the trajectory tracking accuracy and reduce the model complexity [10]. The SMC technique provides a systematic approach that improves the system capability to eliminate the problem of stability preservation [11]. Therefore, due to the characteristics of the sliding mode controller, many researchers have used this control method to overcome the control problem in their systems [12-18].

In this study, a model based sliding mode control method is used for an autonomous mobile in order to get accurate trajectory tracking control approach. Motivated by the advantages of the SMC method, a model based sliding mode control method is proposed and performed on a real mobile robot. In order to illustrate the efficiency of SMC, the real time studies have been realized and compared to the classical PID controller. The main contributions of this study are given as follows; Model based sliding mode controller (SMC) is developed to obtain strong robustness, fast finite-time convergence, precision, and chatter-free control. SMC is utilized to compensate the unknown system dynamics where no knowledge relating to the nonlinear model is available a priori.

The rest of the paper is organized as follows. Section II presents system description of the mobile robot. The model based SMC method for the mobile robot has shown in section III. In section IV, the experimental outcomes have been demonstrated, followed by the conclusion in Section V. 


\section{SYSTEM DESCRIPTION}

Figure 1 shows the designed mobile robot. For the prototype design, only the outer casing and motors of the unmanned vehicle named KOBUKI produced by Turlebot firm were utilized. Instead of the control card, drives, sensors and software used by TurleBot, a unique design has been implemented in this work.

The system has two rear driving wheels and two free caster front wheels. Each driving wheel has been coupled to the shaft of the DC motor and each DC motor is fitted with gearhead and optical encoder. The power for the system is supplied by two $12 \mathrm{~V}$ batteries. Moreover, the system has a personal computer with a controller board for real time applications.

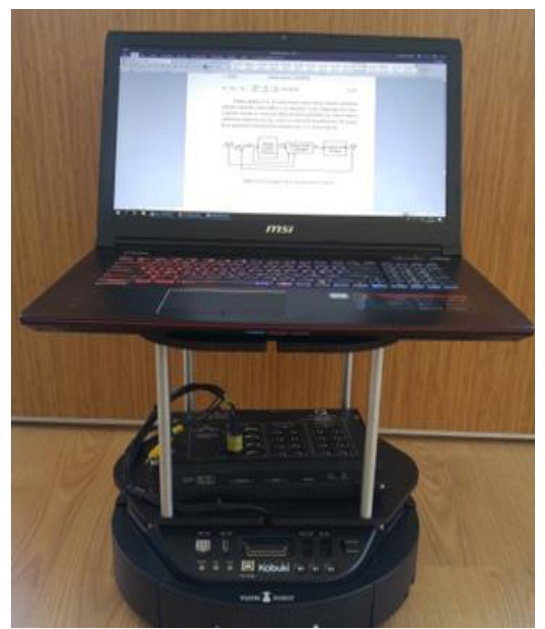

Fig.1. Designed mobile robot

\section{A. Inverse kinematic of mobile robot}

For the mobile robot, inverse kinematics refers to the use of the kinematics equations of the system to determine the angular velocity of the left and the right wheels that provided the desired positon or velocity of the wheelchair. Schematic top view of the mobile robot is shown in Fig. (2). The motion and the orientation of the wheelchair are achieved by independent actuators of the left and right wheels, and each wheel is independent and driven by a DC motor.

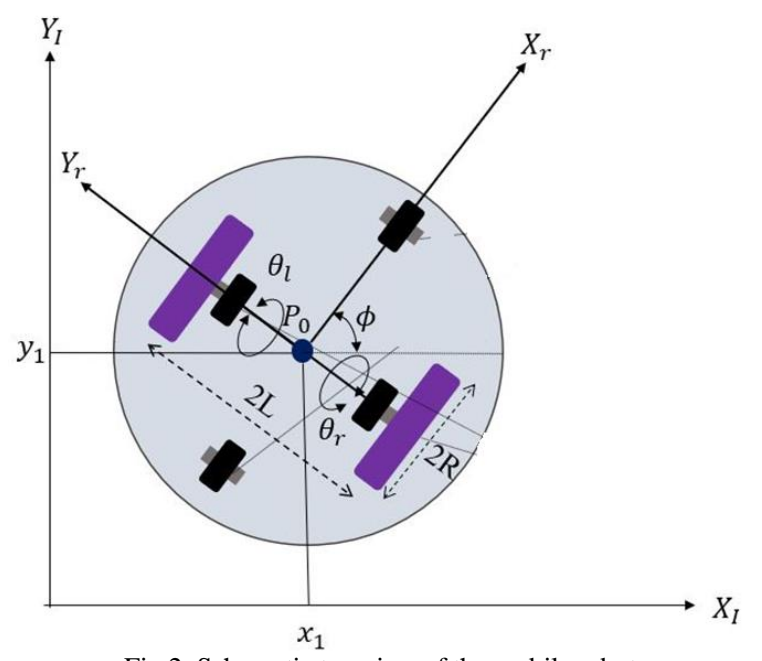

Fig.2. Schematic top view of the mobile robot
For the purpose of analysis, two coordinate systems are defined. The global coordinate system $\left\{X_{I}, Y_{I}\right\}$ is fixed to the Cartesian workspace and the local coordinate system $\left\{X_{r}, Y_{r}\right\}$ is attached to the base of mobile robot platform. The origin of the platform frame is defined to be the midpoint $P_{o}$ on the axis between the wheels. The angular positions of the right and left wheel are given by $\theta_{r}$ and $\theta_{l}$, respectively. The position of any point in the mobile robot can be defined using the transformation of the local coordinate system to the global coordinate system as follows;

$$
X^{I}=R(\phi) X^{r}
$$

where $R(\phi)$ is the orthogonal rotation matrix;

$$
R(\phi)=\left[\begin{array}{ccc}
\cos (\phi) & -\sin (\phi) & 0 \\
\sin (\phi) & \cos (\phi) & 0 \\
0 & 0 & 1
\end{array}\right]
$$

On the conditions of no lateral slip motion, we can write the following kinematic equations in velocity dimension and mobile robot frame;

$$
v=\frac{v_{r}+v_{l}}{2}=R \frac{\left(\dot{\theta_{r}}+\dot{\theta_{l}}\right)}{2}
$$

and the angular velocity of the mobile robot is;

$$
\dot{\phi}=\frac{v_{r}-v_{l}}{2 L}=R \frac{\left(\dot{\theta_{r}}-\dot{\theta_{l}}\right)}{2}
$$

On the other hand, using the orthogonal rotation matrix mobile robot velocities can be obtained from the global coordinate system as follows;

$$
\left[\begin{array}{c}
\dot{x_{1}} \\
\dot{x_{2}} \\
\dot{\phi}
\end{array}\right]=\left[\begin{array}{cc}
\frac{R}{2} \cos (\phi) & \frac{R}{2} \cos (\phi) \\
\frac{R}{2} \sin (\phi) & \frac{R}{2} \sin (\phi) \\
\frac{R}{2 L} & -\frac{R}{2 L}
\end{array}\right]\left[\begin{array}{c}
\dot{\theta}_{r} \\
\dot{\theta_{l}}
\end{array}\right]
$$

\section{B. Constraint equations of mobile robot}

The mobile robot is a class of benchmark non-holonomic system due to its kinematic constraints. The two main nonholonomic constraint equations related to generalized coordinates are obtained from the two main assumptions and choosing two redundant coordinates, $x_{1}, x_{2}$ and two generalized coordinates, $\theta_{r}, \theta_{l}$. The first assumption is that mobile robot cannot move in a lateral direction. This means that in the robot frame, the velocity of the centre point $P_{o}$ is zero along the lateral axis namely $\dot{y}_{r}=0$. Using the orthogonal rotation matrix $R(\phi)$, the first non-holonomic constraint equations related to velocity in the global coordinate system gives;

$$
\dot{x}_{2} \cos (\phi)-\dot{x}_{1} \sin (\phi)=0
$$


The second assumption is that the two driving wheels pure rolling constraint. This means that each wheel maintains a one contact point $P$ with the ground as shown in Fig. (3).

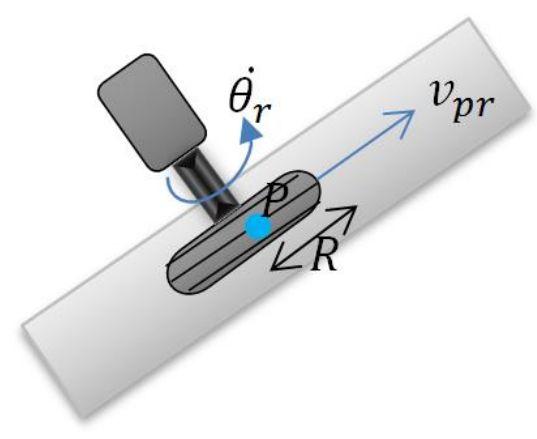

Fig.3. Pure rolling motion constraint

Using the rotation matrix $R(\phi)$, the rolling constraint equations are formulated as follows;

$$
\begin{aligned}
& \dot{x}_{1} \cos (\phi)+\dot{x}_{2} \sin (\phi)+L \dot{\phi}=R \dot{\theta}_{r} \\
& \dot{x}_{1} \cos (\phi)+\dot{x}_{2} \sin (\phi)-L \dot{\phi}=R \dot{\theta}_{l}
\end{aligned}
$$

On the other hand, the mobile robot has a one holonomic constraint equation and to obtain it, first of all we subtract Eq. (7) from Eq. (8) and then integrating with choosing the initial condition;

$$
\begin{gathered}
2 L \dot{\phi}=R\left(\dot{\theta}_{r}-\dot{\theta}_{l}\right) \\
\phi=c\left(\theta_{r}-\theta_{l}\right)
\end{gathered}
$$

where $c$ is equal to $R / 2 L$. For the obtain second non-holonomic constraint equations now we add the Eq. (7) and Eq. (8);

$$
\dot{x}_{1} \cos (\phi)+\dot{x}_{2} \sin (\phi)=\operatorname{cL}\left(\dot{\theta}_{r}+\dot{\theta}_{l}\right)
$$

Hence we may write two non-holonomic constraint equations in matrix form;

$$
A(q) \dot{q}=0
$$

where;

$$
\begin{aligned}
& q=\left[\begin{array}{l}
q_{1} \\
q_{2} \\
q_{3} \\
q_{4}
\end{array}\right]=\left[\begin{array}{l}
x_{1} \\
x_{2} \\
\theta_{r} \\
\theta_{l}
\end{array}\right], A(q)=\left[\begin{array}{llll}
a_{11} & a_{12} & a_{13} & a_{14} \\
a_{21} & a_{22} & a_{23} & a_{24}
\end{array}\right] \\
& =\left[\begin{array}{cccc}
-\sin (\phi) & \cos (\phi) & 0 & 0 \\
-\cos (\phi) & -\sin (\phi) & c L & c L
\end{array}\right]
\end{aligned}
$$

\section{Dynamic modelling of the mobile robot}

The complete dynamic model of the mobile robot which describe the actuating motor torques are derived using Lagrangian approach. The first type of Lagrange's equations will be employed by using the generalized coordinates as $q=$ $\left[x_{1}, x_{2}, \theta_{r}, \theta_{l}\right]^{T}$. For the examined robotic system case, the
Lagrangian equations of the first type can be written as given in Eq. 14.

$$
\frac{d}{d t}\left(\frac{\partial L}{\partial \dot{q}_{l}}\right)-\frac{\partial L}{\partial q_{i}}=Q_{i}-a_{1 i} \lambda_{1}-a_{2 i} \lambda_{2} \text { for } i=1 \text { to } 4
$$

where $Q_{i}$ denotes the external Lagrange force, $\lambda_{1}$ and $\lambda_{2}$ are the Lagrangian multipliers and $L$ is the Lagrangian function.

The total kinetic energy of the mobile robot platform and two wheels can be denoted as follows;

$$
\begin{aligned}
K=\frac{1}{2}\left(m_{c}+2 m_{w}\right) & \left(\dot{x}_{1}^{2}+{\dot{x_{2}}}^{2}\right) \\
& +m_{c} c L\left(\dot{\theta_{r}}-\dot{\theta_{l}}\right)\left(\dot{x_{2}} \cos (\phi)\right. \\
& \left.-\dot{x_{1}} \sin (\phi)\right)+\frac{1}{2} I_{w}\left(\dot{\theta}_{r}{ }^{2}+\dot{\theta}_{l}{ }^{2}\right) \\
& +\frac{1}{2}\left(I_{c}+2 m_{w} L^{2}\right. \\
& \left.+2 I_{m}\right) c^{2}\left(\dot{\theta}_{r}-\dot{\theta}_{l}\right)^{2}
\end{aligned}
$$

where, $m_{c}$ is the mass of the mobile robot without the driving wheels and actuators (DC motors), $m_{w}$ is the mass of each driving wheel (with actuator), $I_{c}$ is the moment of inertia of the mobile robot about the vertical axis through the centre of mass, $I_{w}$ is the moment of inertia of each driving wheel with a motor about the wheel axis, and $I_{m}$ is the moment of inertia of each driving wheel with a motor about the wheel diameter. Using Eq. (15) along with the Lagrangian function, $\mathrm{L}=\mathrm{K}$ the equations of motion of the mobile robot are given by;

$$
\begin{gathered}
\left(m_{c}+2 m_{w}\right) \ddot{x}_{1}-m_{c} d\left(\ddot{\phi} \sin \phi+\dot{\phi}^{2} \cos \phi\right)= \\
\lambda_{1} \sin \phi+\lambda_{2} \cos \phi \\
\left(m_{c}+2 m_{w}\right) \ddot{x_{2}}+m_{c} d\left(\ddot{\phi} \cos \phi-\dot{\phi}^{2} \sin \phi\right) \\
=-\lambda_{1} \cos \phi+\lambda_{2} \sin \phi \\
m_{c} c d\left(\ddot{x}_{2} \cos \phi-\ddot{x}_{1} \sin \phi\right) \\
+\left(\left(I_{c}+2 m_{w} L^{2}+2 I_{m}\right) c^{2}\right. \\
\left.+I_{w}\right) \ddot{\theta}_{r} \\
-\left(I_{c}+2 m_{w} L^{2}+2 I_{m}\right) c^{2} \ddot{\theta}_{l} \\
=\tau_{1}-c L \lambda_{2} \\
-m_{c} c d\left(\ddot{x}_{2} \cos \phi\right. \\
\left.-\ddot{x}_{1} \sin \phi\right) \\
-\left(I_{c}+2 m_{w} L^{2}+2 I_{m}\right) c^{2} \ddot{\theta}_{r} \\
-\left(I_{c}+2 m_{w} L^{2}+2 I_{m}\right) c^{2} \ddot{\theta}_{l} \\
=\tau_{2}-c L \lambda_{2}
\end{gathered}
$$

Hence the actuator torques acting on the two wheels of mobile robot can be determined in the standard form as;

$$
M(q) \ddot{q}+C(q, \dot{q}) q=E(q) \tau-A^{T}(q) \lambda
$$

On the other hand, the system described by Eq. (20) is transformed into a state space representation which is more useful for the model based sliding mode control. For this purpose, a $4 \times 2 S(q)$ matrix can be defined as shown in Eq. (21) and its columns are in the null space of $A(q)$ matrix in the two non-holonomic constraint equations i.e., $A(q) S(q)=0$. 


$$
S(q)=\left[\begin{array}{cc}
c L \cos \phi & c L \cos \phi \\
c L \sin \phi & c L \sin \phi \\
1 & 0 \\
0 & 1
\end{array}\right]
$$

From the two non-holonomic constraint equations, the velocity $\dot{q}$ must be in the null space of $A(q)$. Consequently a reduced smooth vector $\eta=\left[\begin{array}{ll}\eta_{1} & \eta_{2}\end{array}\right]^{T}$ can be defined with mathematical relation form in Eq. (22).

$$
\dot{q}=S(q) \eta, \quad \ddot{q}=\dot{S}(q) \eta+S(q) \dot{\eta}
$$

It's obviously the fact that, for the specific choice of $S(q)$ matrix in Eq. (22), smooth vector $\eta$ can be equal to two wheel velocities vector i.e., $\eta=\dot{\theta}=\left[\begin{array}{ll}\dot{\theta}_{r} & \dot{\theta}_{l}\end{array}\right]$. To eliminate the constraint term $\mathrm{A}^{T}(q) \lambda$ in Eq. (20), this equation's both sides must multiply by $S^{T}(q)$ matrix. Hence the new model can be written as follow;

$$
S^{T}(q) M(q) \ddot{q}+S^{T}(q) C(q, \dot{q})=\tau
$$

Eq. (23) can be rewritten as in the standard form;

$$
\tau=M(q) \ddot{q}+N(q, \dot{q})+\tau_{d}
$$

where, $M(q)$ is the mass matrix, $N(q, \dot{q})$ is the sum of the centrifugal, Coriolis and gravity terms, $\tau_{d}$ is disturbance and friction component of directly related to mobile robot system using a complete dynamic model of systems, respectively. On the other hand Eq. (24) shows that the mobile robot dynamics are expressed as a function of the right and left wheel angular velocities $\dot{\theta}=\left[\begin{array}{ll}\dot{\theta}_{r} & \dot{\theta}_{l}\end{array}\right]$, and the driving motor torques $\tau=$ $\left[\begin{array}{ll}\tau_{r} & \tau_{l}\end{array}\right]^{T}$. As it is seen from Eq. (24), the desired tracking control of the mobile robot can be achieved by changing or controlling the supply voltage of the DC motors.

If the both sides of Eq. 24 is multiplied by $M(q)^{-1}, \ddot{q}$ can be defined as follows;

$$
\ddot{q}_{4 x 1}=f(q)_{4 x 1}+g(q)_{4 x 1} u(t)_{4 x 1}+\zeta(t, u(t))_{4 x 1}
$$

where, $\quad f(q)_{4 \times 1}=-M(q)^{-1} H(q, \dot{q}), \quad g(q)_{4 \times 1}=M(q)^{-1}$, $u(t)_{4 \times 1}=\tau$ and $\zeta(t, u(t))_{4 \times 1}$ stands the bounded uncertainties of the system. The aim of the proposed control technique in this study is to control the system variables, $q_{4 \times 1}$, accurately for the given reference trajectory, $q_{d_{4 \times 1}}$. In order to meet the accurate trajectory tracking, the tracking error $e(t)_{4 \times 1}=q_{d_{4 \times 1}}-q_{4 \times 1}$ should be minimized as much as possible.

\section{MODEL B ASED SLIDING MODE CONTROL TECHNIQUE}

The model based sliding mode control method is a variable structure control and it has a robust structure against the uncertainties in the system and disturbance effects. The control signal used in the sliding mode control method consists of two components. The first component is the equivalent control component and it can be obtained using a sliding surface function with an approximate model of the system. The second component is a switched control component it can be used to compensate the uncertainties affecting the system.

In this proposed method, a sliding surface $\boldsymbol{s}$ is selected as follows;

$$
s=\lambda e+\dot{e}
$$

where, $\lambda$ is a positive constant matrix, $\boldsymbol{e}$ is a tracking error matrix and it can be given as $\boldsymbol{e}=\left[\boldsymbol{q}_{\boldsymbol{d}}-\boldsymbol{q}\right]$. Differentiating Eq. (26) with respect to time, the following equation is obtained

$$
\dot{s}=\lambda \dot{e}+\ddot{e}
$$

This can be further written as;

$$
\dot{\boldsymbol{s}}=\lambda \dot{e}+\left(\ddot{\boldsymbol{q}}_{d}-\ddot{\boldsymbol{q}}\right)
$$

Substituting $\ddot{\boldsymbol{q}}$ from Eq. (25) into Eq. (28) yields;

$$
\dot{s}=\lambda \dot{e}+\ddot{q}_{d}-f(q)-g(q) u
$$

It is well known that, in the second order sliding surface condition, if, $\boldsymbol{s}(\boldsymbol{t})$ and $\dot{\boldsymbol{s}}(\boldsymbol{t})$ equal to null then the tracking error $\boldsymbol{e}$ reaches to zero. Hence, the reaching phase control law $\boldsymbol{u}_{\boldsymbol{e q}}$ can be obtained by using the $\dot{\boldsymbol{s}}(\boldsymbol{t})=\mathbf{0}$ as follows;

$$
u_{e q}=\frac{-f(q)}{g(q)}+\frac{\lambda \dot{e}}{g(q)}+\frac{\ddot{q}_{d}}{g(q)}
$$

In addition to this, it is not appropriate to use only the reaching phase control law to control the system. As mentioned above, the effect of the uncertainties which are constrained but unknown, can be serious for system. Therefore, a switching control law, $\boldsymbol{u}_{\boldsymbol{s c}}$, in Eq. (31), should be added to control signal to ensure the robustness of system against the external or system disturbances.

$$
u_{s c}=k_{x} \operatorname{sgn}(s)
$$

where, $\boldsymbol{k}_{\boldsymbol{x}}$ is the switching gain matrix and $\operatorname{sgn}(\boldsymbol{s})$ can be expressed given as below.

$$
\operatorname{sgn}(s)=\left\{\begin{array}{c}
1 \rightarrow s>0 \\
0 \rightarrow s=0 \\
-1 \rightarrow s<0
\end{array}\right\}
$$

Thus, the total feedback SMC control law $(\boldsymbol{u})$ for the system is written as follows;

$$
\begin{aligned}
u=u_{e q}+u_{s c}= & \frac{-f(q)}{g(q)}+\frac{\lambda \dot{e}}{g(q)}+\frac{\ddot{q}_{d}}{g(q)} \\
& +k_{x} \operatorname{sgn}(s)
\end{aligned}
$$

Practically, the control law given in Eq. (33) can caused oscillations which is called chattering factor. To overcome the control-chattering during the implementation, the sgn (highfrequency switching function), can be approximated to the sat function, which is called the smooth limited function. The 
model based SMC implementation block diagram is shown in Fig. (4).

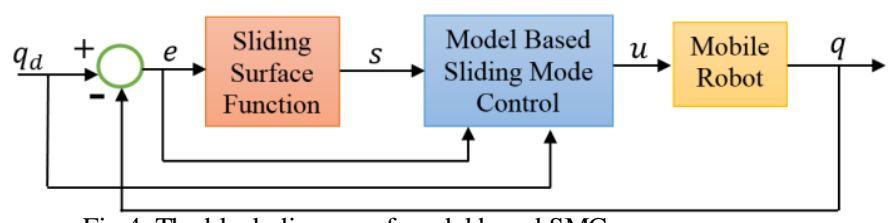

Fig.4. The block diagram of model based SMC

\section{EXPERIMENTAL RESULTS AND DISCUSSIONS}

After modelling the wheeled mobile robot and the proposed SMC controllers, this test platform has simulated experimentally through two different case studies to verify the effectiveness and performance of the proposed methodology with respect to the classical PID control for given task. The controllers have been performed using SIMULINK 2014 from MathWorks. The Q8 USB data acquisition device from Quanser has been used to execute the experiments. In experimental study, the sampling time has been adjusted in $0.001 \mathrm{sec}$. The controller parameters have been adjust as $k_{p}=6, k_{i}=2, k_{d}=$ 0,2 and $\lambda=48,8664, k_{x}=214$ for PID and sliding mode control, respectively.

The controller's performance is tested in the first experiment with an eight shape reference with radius $R=1 \mathrm{~m}$ given by Eq. (34). Since this defined trajectory contains mathematically trigonometric functions, it is necessary for the mobile robot to keep pace with suddenly changing angular speed changes of the right and left wheels of the vehicle in order to be able to track this trajectory.

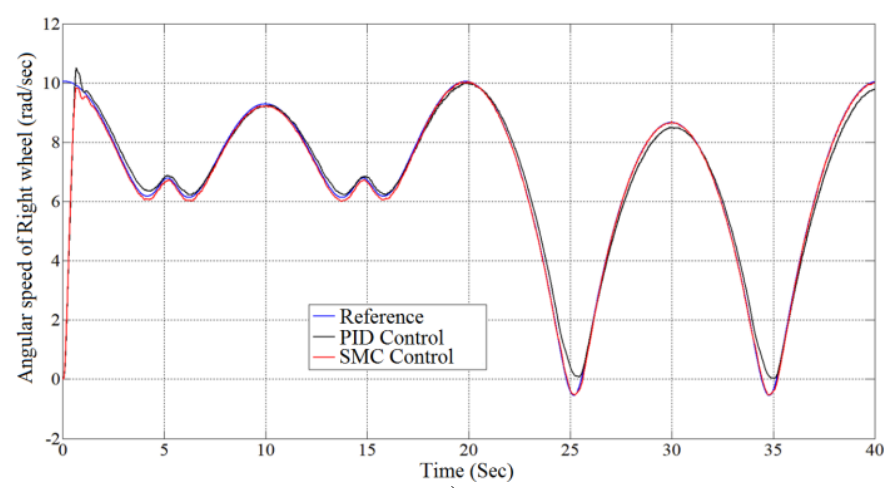

a)

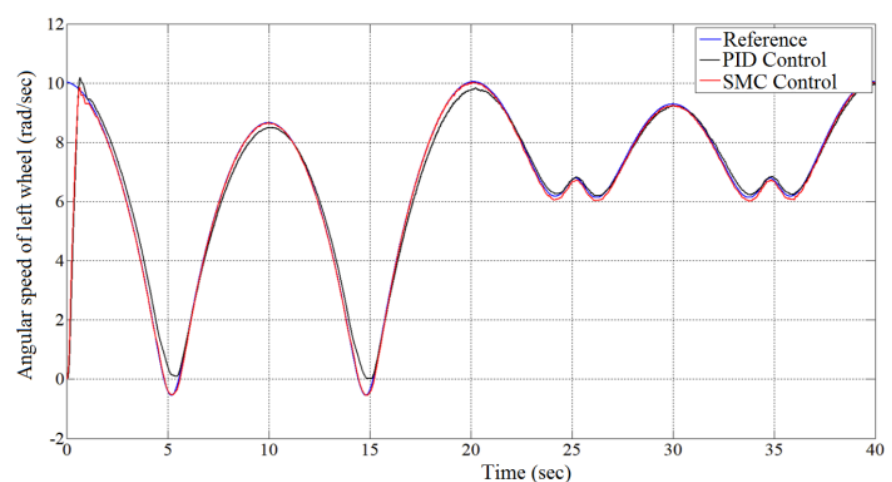

b)

Fig.5. Trajectory tracking responses of right and left wheels

$$
x r=R * \sin \left(2 * \frac{p i}{20} * t\right), y r=R * \sin \left(\frac{p i}{20} * t\right)
$$

Using the kinematic equations obtained in Section II, the angular velocity changes that should be realized by the right and left wheels for mobile robot to follow this defined trajectory and the performances of the PID and SMC controllers have been shown in Fig. (5). And also, Fig. (6) presents the tracking errors of the both controllers.

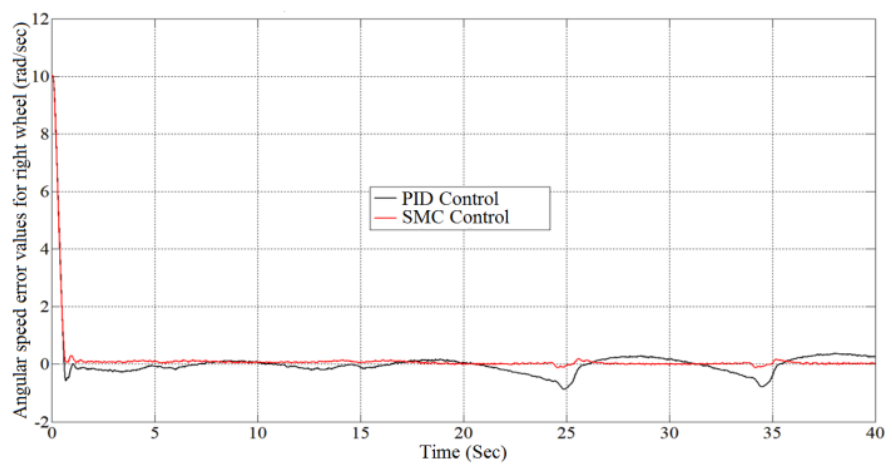

a)

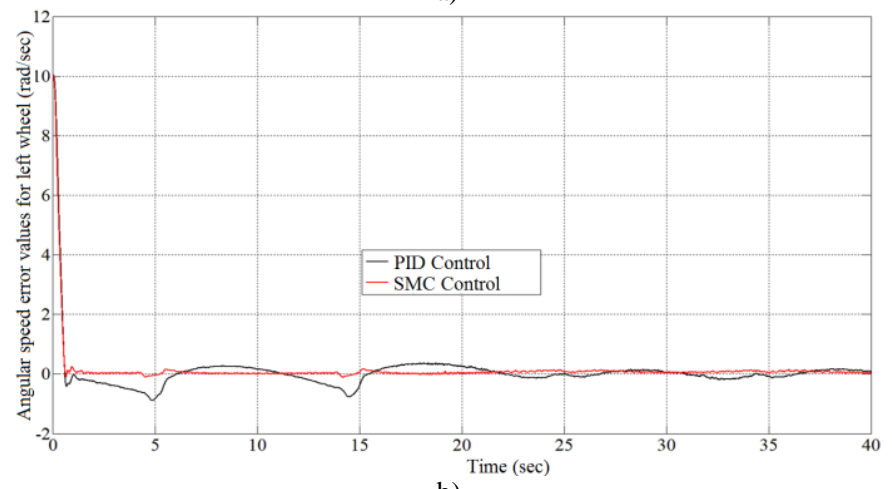

b)

Fig.6. Angular speed error values of right and left wheels

As it can be seen from Figs. (5) and (6), the proposed SMC technique follows the reference trajectory more accurately than the PID control technique. According to the trajectory tracking response for both controllers in Figs. (5) and (6), PID controller causes overshoot values at the beginning of the motion. Meanwhile, with the robust control designs, the SMC improved the control performances with quite small tracking errors. By employing the system dynamic model to SMC controller, the controller makes the system more robust against the presence of uncertainties and disturbances in the feedback loop. However, as can be seen from figures, overshoot values are eliminated completely for the proposed SMC controller case due to the SMC technique is a robust and an efficient control technique for un-modelled dynamics and un-known loads of the robotic system. Also, both transient and steady-state error values are reduced by using the proposed SMC controller as shown in Figures.

\section{CONCLUSION}

In this study, the model based SMC has been proposed in order to control the uncertain nonlinear mobile robotic system. In order to get finite-time convergence, better tracking of 
trajectory and chatter-free control, a model based SMC is developed. The experimental outcomes strongly verified that the proposed SMC provides a quite well trajectory tracking performance during the motion. Owing to the proposed algorithm, the SMC controller follows the reference with small error when it is compared with the responses of classical PID controller. For future work, the authors aim to design adaptive sliding mode control strategy by using the same model based method for the considered mobile robot system. Using the adaptive SMC in the proposed controller, it is expected that the trajectory tracking error value will be remarkably reduced.

\section{REFERENCES}

[1] N. Uchiyama, T. Hashimoto, S. Sano and S. Takagi, "Model-Reference Control Approach to Obstacle Avoidance for a Human-Operated Mobile Robot," in IEEE Transactions on Industrial Electronics, vol. 56, no. 10, pp. 3892-3896, Oct. 2009.

[2] M. Makarov, M. Grossard, P. Rodríguez-Ayerbe and D. Dumur, "Modeling and Preview\$H_linfty\$Control Design for Motion Control of Elastic-Joint Robots With Uncertainties," in IEEE Transactions on Industrial Electronics, vol. 63, no. 10, pp. 6429-6438, Oct. 2016.

[3] S. Li, Y. Zhang and L. Jin, "Kinematic Control of Redundant Manipulators Using Neural Networks," in IEEE Transactions on Neural Networks and Learning Systems, vol. 28, no. 10, pp. 2243-2254, Oct. 2017.

[4] Y. Zhang, S. Chen, S. Li and Z. Zhang, "Adaptive Projection Neural Network for Kinematic Control of Redundant Manipulators With Unknown Physical Parameters," in IEEE Transactions on Industrial Electronics, vol. 65, no. 6, pp. 4909-4920, June 2018.

[5] Z. Hou, A. Zou, L. Cheng and M. Tan, "Adaptive Control of an Electrically Driven Nonholonomic Mobile Robot via Backstepping and Fuzzy Approach," in IEEE Transactions on Control Systems Technology, vol. 17, no. 4, pp. 803-815, July 2009.

[6] D. Chwa, "Fuzzy Adaptive Tracking Control of Wheeled Mobile Robots With State-Dependent Kinematic and Dynamic Disturbances," in IEEE Transactions on Fuzzy Systems, vol. 20, no. 3, pp. 587-593, June 2012.

[7] A. Rojas-Moreno and G. Perez-Valenzuela, "Fractional order tracking control of a wheeled mobile robot," 2017 IEEE XXIV International Conference on Electronics, Electrical Engineering and Computing (INTERCON), Cusco, 2017, pp. 1-4.

[8] Y. Zhao, N. Chen and Y. Tai, "Trajectory tracking control of wheeled mobile robot based on fractional order backstepping," 2016 Chinese Control and Decision Conference (CCDC), Yinchuan, 2016, pp. 67306734.

[9] T. Chen, B. Dai, J. Song and L. Zhou, "On lateral fractional order controller in auto-parking system," Proceedings of the 30th Chinese Control Conference, Yantai, 2011, pp. 3429-3434.

[10] V. Utkin, "Variable structure systems with sliding modes," in IEEE Transactions on Automatic Control, vol. 22, no. 2, pp. 212-222, April 1977.

[11] C. -. Su, T. -. Leung and Y. Stepanenko, "Real-time implementation of regressor-based sliding mode control algorithm for robotic manipulators," in IEEE Transactions on Industrial Electronics, vol. 40, no. 1, pp. 71-79, Feb. 1993.
[12] L. M. Capisani and A. Ferrara, "Trajectory Planning and Second-Order Sliding Mode Motion/Interaction Control for Robot Manipulators in Unknown Environments," in IEEE Transactions on Industrial Electronics, vol. 59, no. 8, pp. 3189-3198, Aug. 2012.

[13] J. Baek, M. Jin and S. Han, "A New Adaptive Sliding-Mode Control Scheme for Application to Robot Manipulators," in IEEE Transactions on Industrial Electronics, vol. 63, no. 6, pp. 3628-3637, June 2016.

[14] F. Zhang, "High-speed nonsingular terminal switched sliding mode control of robot manipulators," in IEEE/CAA Journal of Automatica Sinica, vol. 4, no. 4, pp. 775-781, 2017.

[15] M. Van, S. S. Ge and H. Ren, "Finite Time Fault Tolerant Control for Robot Manipulators Using Time Delay Estimation and Continuous Nonsingular Fast Terminal Sliding Mode Control," in IEEE Transactions on Cybernetics, vol. 47, no. 7, pp. 1681-1693, July 2017.

[16] J. Lee, P. H. Chang and M. Jin, "Adaptive Integral Sliding Mode Control With Time-Delay Estimation for Robot Manipulators," in IEEE Transactions on Industrial Electronics, vol. 64, no. 8, pp. 6796-6804, Aug. 2017.

[17] G. P. Incremona, M. Rubagotti and A. Ferrara, "Sliding Mode Control of Constrained Nonlinear Systems," in IEEE Transactions on Automatic Control, vol. 62, no. 6, pp. 2965-2972, June 2017.

[18] A. Ferrara and G. P. Incremona, "Design of an Integral Suboptimal Second-Order Sliding Mode Controller for the Robust Motion Control of Robot Manipulators," in IEEE Transactions on Control Systems Technology, vol. 23, no. 6, pp. 2316-2325, Nov. 2015.

\section{BIOGRAPHIES}

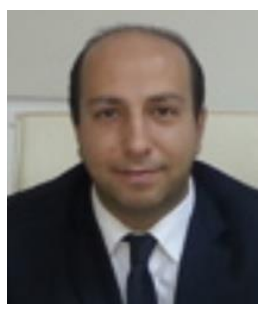

AHMET DUMLU was born in Erzurum, Turkey, in 1980. He received the B.S. degree in electronic engineering from Erciyes University, Kayseri, Turkey, in 2004, the M.S. degree in electrical \& electronic engineering from Ataturk University, Erzurum, in 2009, and the Ph.D. degree in electrical engineering from Ataturk University, Erzurum, Turkey, in 2003. He is currently an Associate Professor with the Department of Electrical and Electronics Engineering, College of Engineering, Erzurum Technical University, Erzurum. His work has focused on the development and application of control theory to a variety of mechatronic systems, with a focus on observation and estimation-based control. Much of his recent work has focused on the analysis and control of dynamical systems that arise in engineering applications. His areas of interest also include fuzzy logic and fuzzy control, neural networks, genetic algorithms, and their applications to dynamic systems and electrical engineering.

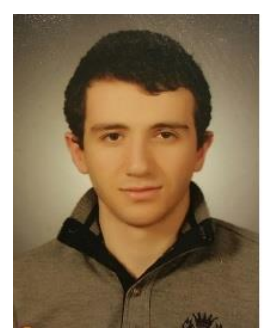

MEHMET RASIM YILDIRIM was born in Erzurum, Turkey, in 1990. He received the B.S. degree from Ataturk University, Erzurum, Turkey, in 2013 and he is currently working toward the M.S. degree. His research interests include the theory of mechatronic and robotic systems, with a focus on observation and estimation-based control. 